\title{
Influence of elastic strains on the mask ratio in glassy polymer nanoimprint
}

\author{
Graham L. W. Cross, ${ }^{a)}$ Barry S. O'Connell, and John B. Pethica \\ SFI Trinity Nanoscience Laboratory, Trinity College, Dublin 2, Ireland
}

(Received 22 June 2004; accepted 28 December 2004; published online 14 February 2005)

\begin{abstract}
During glassy polymer nanoimprint, a supported film is extruded from protruding (punch) to recessed (cavity) regions of a patterned stamp. The completeness of this extrusion determines the mask ratio for lithographic applications. We show that, for a given punch contact size, there is a residual layer of unextruded material with a mean thickness that is independent of initial film thickness, stamping time, or applied maximum load. Depth sensing indentation enables us to monitor deformation during the imprinting as well as after, and so understand the deformation process involved. It is found that both the geometry and mean thickness of the residual layer are influenced by the overall elastic properties of the stamping system. (C) 2005 American Institute of Physics. [DOI: 10.1063/1.1868074]
\end{abstract}

Nanoimprint lithography is an emerging nanofabrication technique that enables both high resolution and rapid throughput. Variations based on solid phase cutting and stamping, ${ }^{1,2}$ hot embossing, ${ }^{3-5}$ or liquid molding ${ }^{6,7}$ have been reported. All techniques share the common requirement that during the forming process, sufficient sample material flow must ensue to guarantee pattern transfer fidelity, while all other parasitic deformation (such as that arising from demolding stresses) must be minimized. Underlying this requirement is the physical principle that sufficient amounts of shear stress must be generated in the correct locations to give the plastic strain and thus the shape change required. This is achieved in the hot embossing and capillary molding strategies via the minimal shear strength of near-liquid-state materials. In the case of solid phase forming, yield-inducing shearing must be maintained to sustain plastic flow until reproduction is achieved. This approach therefore requires larger stress generation and so can introduce significant elastic strains. However, it has the potential advantages of forming a wider range of materials, of not requiring temperature (and hence dimensional) changes during molding, and suffering less from demolding adhesion problems.

Imprinting thin films to make a mask involves two related processes: the ability to uniformly extrude material from below punch regions of the stamp and the ability to uniformly fill recessed stamp regions with the extruded material. In this letter we focus on the issue of residual material left between punch areas of the stamp and the substrate. We use a depth sensing nanoindentation system ${ }^{8}$ to record the forces and displacements of a flat punch as it is driven into and then withdrawn from a polymer film. This gives a continuous readout of the overall forces and strains during all stages of the imprint process, and hence additional information beyond simply imaging the residual imprints.

The initial geometry is shown in Fig. 1. A flat punch of diameter $7400 \mathrm{~nm}$ and height $2200 \mathrm{~nm}$ was fabricated by focused ion beam (FIB) milling the surface of a $1 \mathrm{~mm}$, polished, single crystal silicon sphere (Silicon Ball Corp.). The punch surface roughness was less than $3 \mathrm{~nm}$ according to atomic force microscopy (AFM); it was noted that these residual polishing scratches were replicated in the polymer

\footnotetext{
${ }^{a)}$ Electronic mail: graham.cross@tcd.ie
}

when indenting. The polymer used was polyvinyl acetate (PVAc) (Aldrich Corp.) of $M_{n}=12$ 800, a degree of polymerization much less than the critical entanglement backbone chain weight. ${ }^{9}$ The bulk $T_{g}$ was $35^{\circ} \mathrm{C}$. Samples consisting of films of PVAc on cleaned silicon wafer substrates (110 orientation) were prepared by $4000 \mathrm{rpm}$ spin casting from toluene solutions of $7,10,15$, and $20 \mathrm{mM}$ concentration, yielding films of thickness 500, 900, 1310, and $2200 \mathrm{~nm}$, respectively. In addition, a bulk sample was prepared by squeezing a pellet of PVAc between two silicon wafer chips at $130{ }^{\circ} \mathrm{C}$. All samples were annealed at $100{ }^{\circ} \mathrm{C}$ for $60 \mathrm{~min}$ on a hot plate. During experiments, relative humidity was $30 \% \pm 15 \%$, and temperature was $23 \pm 1{ }^{\circ} \mathrm{C}$.

AC load modulation ( $3 \mathrm{~nm}$ at $45 \mathrm{~Hz}$ ) was used to measure contact stiffness continously during all indentations. ${ }^{10}$ For the bulk sample we can use the standard expression for contact stiffness $S=2 a E /\left(1-\nu^{2}\right),{ }^{11}$ where $a$ is the punch radius, $\nu=0.35$ the Poisson ratio, and $E$ the Young's modulus. We found $E=4.2 \pm 0.1 \mathrm{GPa}$ at initial contact, increasing to $5.0 \pm 0.1 \mathrm{GPa}$ at $2200 \mathrm{~nm}$ depth. This increase may be evidence of some strain induced elastic anisotropy ${ }^{12}$ but may also be due to effects of polymer friction on the punch sidewell. In separate nanoindentation tests on the samples using a Berkovich (pyramidal) indenter ${ }^{8}$ we measured the hardness (mean contact pressure) to be $200 \pm 10 \mathrm{MPa}$. As might be expected for a low molecular weight polymer, there was no evidence for strain hardening in these pyramidal indentations.
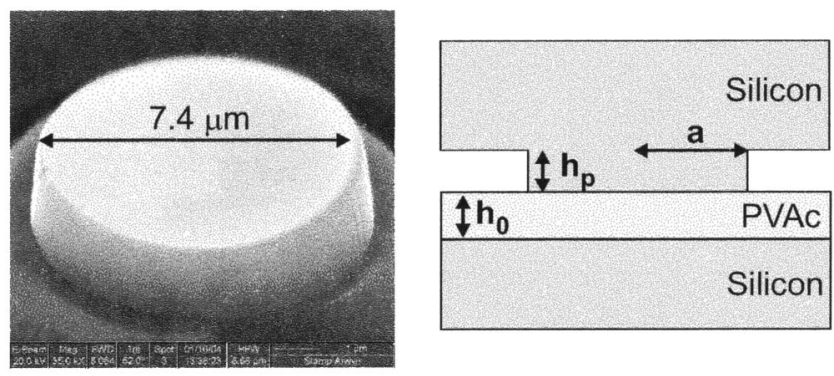

FIG. 1. SEM image of the FIB milled silicon flat punch and schematic of the preindentation geometry. A PVAc polymer film of thickness $h_{0}$ is indented by a punch of radius $a$ and height $h_{p}$. 

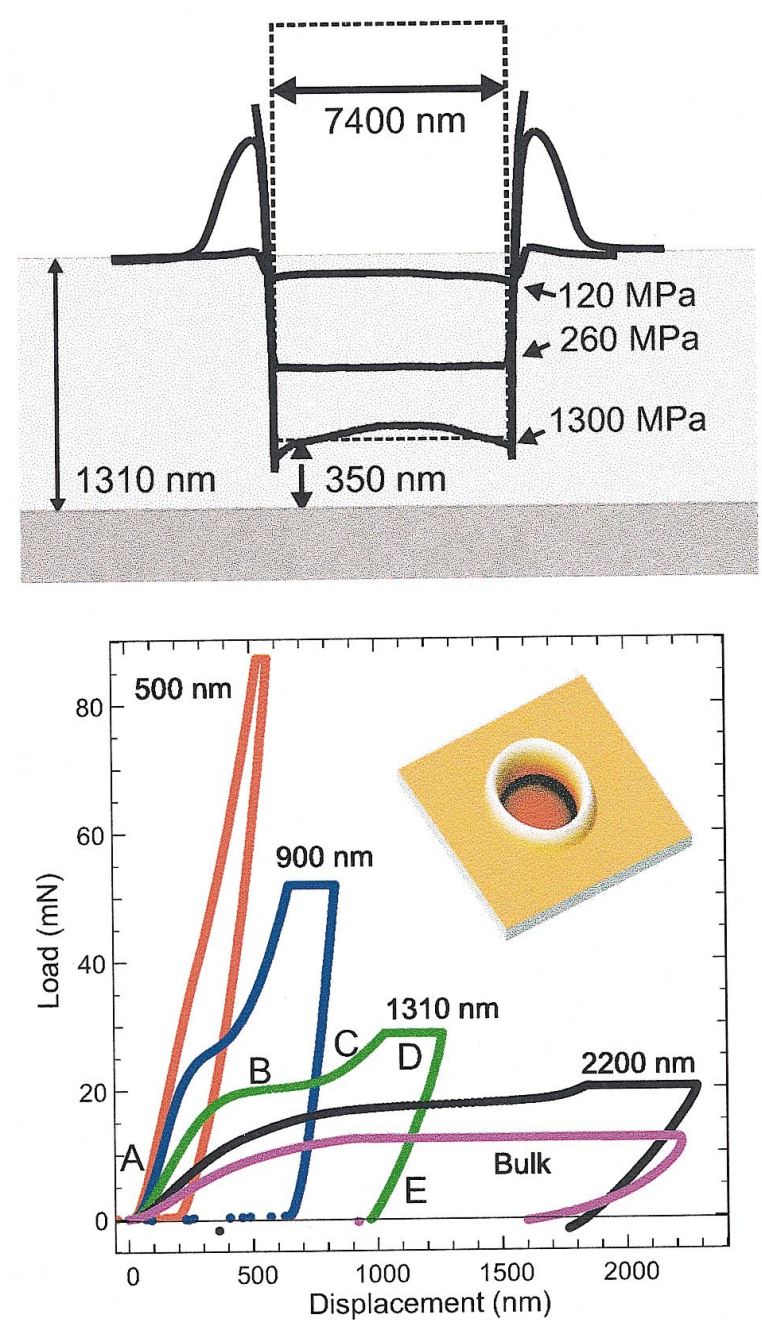

FIG. 2. (Color) (Upper) AFM cross-section profiles of residual imprints in a $1310 \mathrm{~nm}$ thick film. Overlay is punch position at surface separation for deepest indent. Mean pressure is the maximum load divided by punch area. (Lower): punch indentation load vs displacement curves for PVAc bulk and thin films.

A typical stamping experiment had the following sequence of events: indentation to defined fractions of the film thickness at a load rate of $0.8 \mathrm{mN} / \mathrm{s}$, holding the peak load for $600 \mathrm{~s}$, and finally unloading at $0.5 \mathrm{mN} / \mathrm{s}$. The residual indent shapes after indentation were measured by intermittent contract mode AFM. Standard AFM tips were found to introduce significant image distortions. Therefore, special, high aspect ratio tips, of opening angle $25^{\circ}$ and $3.5 \mu \mathrm{m}$ length, were prepared in the FIB and used for all imaging.

Complete load-displacement curves for the punch into each film are shown in Fig. 2. All samples show certain common characteristics, in five successive regimes indicated by $\mathrm{A}-\mathrm{E}$ on the $1310 \mathrm{~nm}$ film data. (A) An initial reversible elastic loading; (B) a relatively sudden turn over to plastic flow at almost constant load and hence mean pressure; (C) in the case of film samples, a rising load due to the increasing influence of the hard silicon substrate; (D) a slow creep flow whilst the maximum load is held; and (E) elastic recovery during unloading. Qualitatively similar behavior was observed for loading rates varying over three orders of magnitude. The final unloading (demolding) shows only very small adhesive forces at separation.

AFM images were taken $<60$ min after indentation. Note the central flat depression and the surrounding raised Downloaded 09 Mar 2009 to 134.226.1.229. Redistribution subject

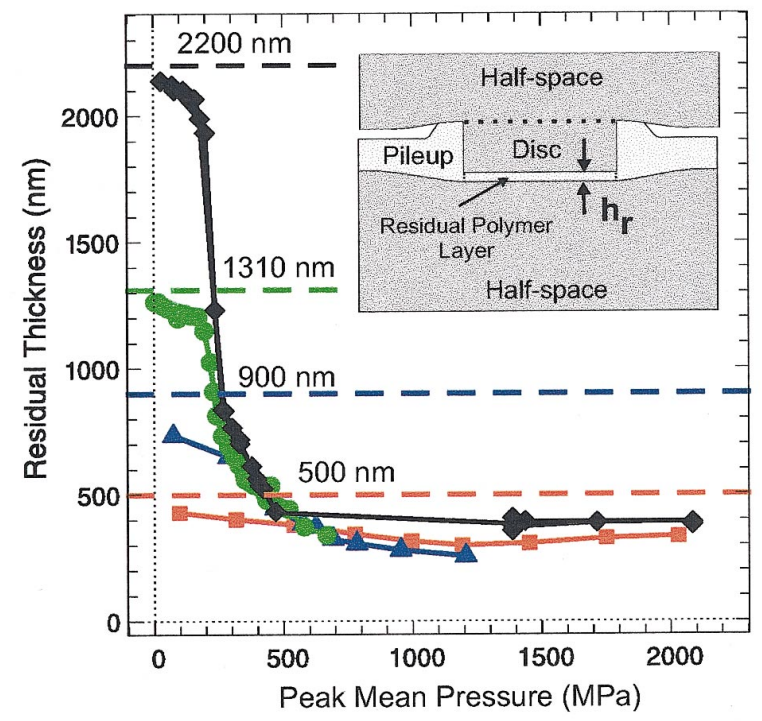

FIG. 3. (Color) Residual polymer thickness after indentation as a function of peak mean punching pressure for the four films with initial thickness indicated. A terminal thickness of about $350 \mathrm{~nm}$ remains regardless of initial film thickness or peak pressure applied. The inset shows a schematic of the indented system at peak load and the components of a four spring contact representation discussed.

ring of extruded polymer around the punch edge (typical image in Fig. 2). Imaged residual indent depths agree with depths deduced from punch displacement at the end of unloading. AFM cross-section profiles are shown in the upper portion of Fig. 2 for three different indents into the $1310 \mathrm{~nm}$ film, each of different maximum load. The shallowest indent only reaches regime (A) at $120 \mathrm{MPa}$ before unloading; some plasticity occurs at the punch edge, giving a shallow convex net shape. The next indent reaches $260 \mathrm{MPa}$, after full yield has ensued in regime (B); the residual profile has a uniform flat shape. In the third indent a high pressure of $1300 \mathrm{MPa}$ in regime (D) is reached; the net shape is convex, probably due in part to deformation of the punch shape itself, which will be discussed below.

Measured residual film thicknesses under the punch after indentation are shown in Fig. 3 as a function of maximum imprinting pressure applied to the punch. For pressures below the bulk PVAc hardness of $200 \mathrm{MPa}$ there is only slight change from initial film thickness. As the pressure goes over $200 \mathrm{MPa}$, significant plastic deformation occurs and all films thin rapidly with increasing pressure. Above $400-500 \mathrm{MPa}$, however, a terminal residual thickness of $\sim 350 \mathrm{~nm}$ is reached. Note that this thickness does not depend on the initial film thickness; punch pressures exceeding the polymer hardness by a factor of ten have no further plastic effect. Interestingly, the contact stiffness of all indents which reach this terminal thickness is $0.4 \times 10^{6} \mathrm{~N} / \mathrm{m}$ irrespective of starting film thickness.

Post yield, large plastic strains are present and the polymer deformation will resemble squeeze flow of viscoplastic material between two plates, ${ }^{13,14}$ but with constraint at the edges due to the adjoining polymer beyond the squeezed zone. Clearly, as the squeezed film becomes thinner and its aspect ratio becomes greater, the indent load required to generate everywhere the shear (yield) stress for plastic flow rises rapidly. Large, essentially hydrostatic stresses will be developed under the punch. The above results can also be underto AlP license or copyright; see http://apl.aip.org/apl/copyright.jsp 
stood if the elastic strains present in the imprint at high load are considered. A schematic of the components involved is in Fig. 3; the vertical scale is exaggerated for clarity so the edge effects are actually small. If for a moment therefore, we ignore the extruded polymer piled up outside the punch, there are essentially four elements in series: an upper elastic silicon half-space, a cylindrical silicon disk of radius $a$ and height $h_{p}=2200 \mathrm{~nm}$, a cylindrical disk of residual polymer thickness $h_{r}=350 \mathrm{~nm}$, and a lower elastic silicon half-space. To estimate the overall stiffness, first assume there are no frictional tractions between these elements. The stiffness of the silicon disk is $S=\pi a^{2} E_{\mathrm{Si}} / h_{p}=2.9 \times 10^{6} \mathrm{~N} / \mathrm{m}$. The stiffness of the residual polymer film is $\pi a^{2} E_{\text {poly }} / h_{r}=0.6$ $\times 10^{6} \mathrm{~N} / \mathrm{m}$. The stiffness of each silicon half-space is $2 a E_{\mathrm{Si}} /$ $\left(1-v^{2}\right)=1.2 \times 10^{6} \mathrm{~N} / \mathrm{m}$. The total stiffness of the four springs in series is thus $0.27 \times 10^{6} \mathrm{~N} / \mathrm{m}$. This means that of the high load elastic strain, $10 \%$ is in the punch disk, $45 \%$ is in the bounding silicon half-spaces. Turning now to the simplifying assumptions made above, the polymer outside the residual disk will constrain the disk and therefore increase its stiffness. Adding frictional tractions at the interfaces between the elements will also increase the polymer disk stiffness. A noslip boundary gives ${ }^{15}$ stiffness $3 \pi E_{\text {poly }} a^{4} / 2(1+v) h_{r}^{3}=32$ $\times 10^{6} \mathrm{~N} / \mathrm{m}$. In summary, the residual polymer film stiffness is likely to be significantly higher than the $0.6 \times 10^{6} \mathrm{~N} / \mathrm{m}$ estimated above. In turn the estimated total stiffness will be closer to our experimental value of $0.4 \times 10^{6} \mathrm{~N} / \mathrm{m}$. However, the stiffness of the silicon half-spaces is only weakly affected by the assumptions; the strain falls as $1 / r$ into the silicon, so unlike the disks, has a significant far field. ${ }^{11}$ Therefore they become the dominant elements, so the imprint strain at high loads is mostly accommodated by elastic relaxation of the bounding silicon half-spaces and not the continued extrusion of polymer desired.

Deformation of imprinting tools thus cannot be ignored, and punch rigidity limits the ability to imprint larger areas of very thin film. It is not so much a problem for very small area (lower aspect ratio) features, since the half-space rigidity scales with a, while the film stiffness scales with $a^{2}$. We conclude that when scaling imprint down into the nanometer range, one cannot ignore the aspect ratio of features to be printed, since punch materials have finite elastic modulus. Efforts to improve masking contrast of imprinted features by the addition of normal pressure lead instead to a loss of uniformity, with little or no contrast gain. In a recent study of imprint into long chain, glassy polystyrene ${ }^{16}$ it was shown how strain hardening could explain the nonuniformity observed across small sets of imprinted identical lines. Here, due to the material that we have chosen, we do not have this flow complexity. Instead, we see that, for a given local geometry, a terminal thickness is achieved that reflects the stress conditions in the final contact state, and not the history of the preceding flow process. This somewhat surprising result that the extent of extrusion of soft polymer is quite limited is in fact an essential characteristic of the high aspect ratios present in nanoimprint.

${ }^{1}$ N. Stutzmann, T. A. Tervoort, K. Bastiaansen, and P. Smith, Nature (London) 407, 613 (2000).

${ }^{2}$ D. Khang, H. Yoon, and H. Lee, Adv. Mater. (Weinheim, Ger.) 13, 749 (2001).

${ }^{3}$ S. Y. Chou, P. R. Krauss, and P. J. Renstrom, J. Vac. Sci. Technol. B 14, 4129 (1996).

${ }^{4}$ H. Schift and L. J. Heyderman, Nanorheology. Squeeze Flow in Hot Embossing of Thin Films (Kluwer Academic/Plenum, Amsterdam, 2003), Chap. 3.

${ }^{5}$ H. C. Scheer and H. Schulz, Microelectron. Eng. 56, 311 (2001).

${ }^{6}$ M. Colburn, S. Johnson, M. Stewart, S. Damle, T. Bailey, B. Choi, M. Wedlake, T. Michaelson, S. V. Sreenivasan, J. Ekerdt, and C. G. Willson, Proc. SPIE 3676, 379 (1999).

${ }^{7}$ Y. Xia and G. M. Whitesides, Annu. Rev. Mater. Sci. 28, 153 (1998).

${ }^{8}$ J. B. Pethica, R. Hutchings, and W. C. Oliver, Philos. Mag. A 48, 593 (1983).

${ }^{9}$ L. H. Sperling, Introduction to Physical Polymer Science (Wiley, New York, 2001).

${ }^{10}$ J. B. Pethica and W. C. Oliver, Mater. Res. Soc. Symp. Proc. 130, 13 (1989).

${ }^{11}$ K. L. Johnson, Contact Mechanics (Oxford University Press, London, 1985).

${ }^{12}$ I. M. Ward and D. W. Hadley, An Introduction to the Mechanical Properties of Solid Polymers (Wiley, New York, 1993).

${ }^{13}$ M. J. Adams, I. Aydin, B. J. Briscoe, and S. K. Sinha, J. Non-Newtonian Fluid Mech. 71, 41 (1997).

${ }^{14}$ D. N. Smyrnaios and J. A. Tsamopoulos, J. Non-Newtonian Fluid Mech. 100, 165 (2001).

${ }^{15}$ F. Yang, Mech. Mater. 30, 275 (1998).

${ }^{16}$ P. Hong and H. Lee, Appl. Phys. Lett. 83, 2441 (2003). 